\title{
Poor diet reflected in height, weight of low-income Hispanics
}

\author{
Lorrene D. Ritchie
}

Cathi L. Lamp \ Joanne P. Ikeda

\author{
Suzanne P. Murphy
}

a

Olivier Receveur

\begin{abstract}
A study of low-income Hispanic women and children in California revealed a high prevalence of individuals who were short in stature and overweight. Children in San Jose tended to be shorter than their Tulare County counterparts. Even after adjusting for socioeconomic variables and hereditary differences, the difference in size remained significant. These findings indicate that low-income Hispanics might benefit from nutrition and health interventions.
\end{abstract}

Hispanics are the second largest and fastest growing minority in the United States. In 1987, 60\% of all Hispanics in the United States lived in California. Many of them live in poverty; in California, the poverty rate is higher for Hispanics than for non-Hispanics. Despite this fact, few studies have examined factors influencing the nutritional status of California's Hispanic population, and very little is known about the potential differences between rural and urban groups. Yet, if the nutritional problems of urban and rural Hispanics are found to be different, public health measures can be more appropriately targeted.

The objective of this study was to examine dietary factors and nutritional status of two low-income populations of Hispanic women and their children. This paper reports the findings on status as indicated by height and weight. The study was conducted in 1989-90, using a pool of randomly recruited, low-income Hispanic families from rural Tulare County and San Jose, California. The families were re- cruited primarily from clinics of the Special Supplemental Food Program for Women, Infants, and Children (WIC), which is a federally funded program targeting low-income pregnant and postpartum women and to children up to 5 years old who are at nutritional risk.

Socioeconomic status was established by gathering information from the mother on factors such as income, education and housing. Trained nutritionists measured the height and weight of the mother and at least two of her children between the ages of 1 and 9 . The measurements were made without shoes and with light clothing. Pregnant women and women with a child less than three months of age were excluded from analyses. The sample included 95 mothers with 226 children in Tulare, and 57 mothers with 124 children in San Jose. All of the Tulare participants were from farmworker families.

\section{Family characteristics}

Household information. Both the farmworker and urban samples con- sisted primarily of very low-income Mexican-American families (table 1). Compared to 1987 national statistics for Hispanics, the families in Tulare County and San Jose were larger on average by two persons, with an average household size of six. The majority of mothers were born in Mexico or another Latin American country, although the majority of children were born in the United States.

Unemployment was high, with a jobless rate of $35 \%$ for the farmworker families and $47 \%$ for urban parents. As a consequence, total income was lower than the national average. More than $90 \%$ of families lived below the federal poverty level. Mothers in San Jose had more schooling (7.4 years versus 6.1 years for Tulare mothers, and a higher rate of English-speaking language competency (32\% compared to 19\% for Tulare mothers). At the same time the unemployment rate was higher for San Jose families, and per capita yearly wage was lower, $\$ 1,119$ compared to $\$ 1,457$ for Tulare families. The median family income for Hispanics nationally, including wages and public assis-

\begin{tabular}{|lcc}
\hline TABLE 1. Characteristics of low-income Hispanic households in two California locations \\
\hline \hline & $\begin{array}{c}\text { Tulare County } \\
\text { farmworkers }\end{array}$ & $\begin{array}{c}\text { San Jose } \\
\text { residents }\end{array}$ \\
\hline Household & 35 & 47 \\
$\%$ unemployment & 91 & 95 \\
$\%$ below poverty level & $\$ 1,457$ & $\$ 1,119$ \\
mean per capita yearly wage income & $\$ 753$ & $\$ 1,190$ \\
mean per capita yearly housing cost & 83 & 63 \\
$\%$ two-parent household & 95 & 25 \\
Mothers & 11 & 32 \\
number & 11.6 \\
$\%$ born in United States & 11.4 & 7.4 \\
mean years in United States & 6.1 & 124 \\
mean years of school & 226 & 73 \\
\hline
\end{tabular}


tance, was nearly $\$ 16,500$ in 1988 dollars, which was almost double the $\$ 9,000$ reported in Tulare, and the $\$ 9,900$ in San Jose in 1989-90.

The percentage of two-parent households was fairly similar to the national Hispanic average of $71 \%$, with $83 \%$ in Tulare and $63 \%$ in San Jose. More families in San Jose than Tulare were headed by a single female, which may account for the employment and wage income disparities. Despite having similar total incomes, farmworker families may have been slightly better off financially compared to their urban counterparts in San Jose where the cost of living was substantially higher; for example, per capita yearly housing cost was $\$ 1,190$ in San Jose and $\$ 753$ in Tulare.

Mother's height and weight. The San Jose and Tulare County mothers showed no significant differences in their heights and weights (table 2). However, they were shorter (by about $8 \mathrm{~cm}$ ) and heavier relative to their height when compared to all U.S. women, using reference data from the National Health and Nutrition Examination Survey, 1976-80 (NHANES II). Mean body mass index (BMI, weight divided by height squared) was about $28 \mathrm{~kg} / \mathrm{m}^{2}$ versus the national reference mean of $23.1 \mathrm{~kg} / \mathrm{m}^{2}$. They were also more than $2 \mathrm{~cm}$ shorter than a national sample of Hispanic women from the Hispanic Health and Nutrition Examination Survey, 1982-84 (HHANES). More women were classified as overweight (BMI at or above the 85th percentile of women aged 20-29 years from NHANES II) in Tulare (49\%) and San Jose $(56 \%)$, compared to a national sample of Hispanic women (39\% or less from HHANES).

Few of the variables examined, including income, were found to be predictive of the mother's height. Since height deficiencies are acquired during childhood, present living conditions would not be expected to have a marked effect on adult stature.

Women who had lived in the United States for 10 years or more were taller than more recent immigrants, a finding which might reflect improved living conditions during the childhood growth period.

TABLE 2. Nutrition status indicators of low-income Hispanic women and children in two California locations

\begin{tabular}{lcc}
\hline \hline & $\begin{array}{c}\text { Tulare County } \\
\text { farmworkers }\end{array}$ & $\begin{array}{c}\text { San Jose } \\
\text { residents }\end{array}$ \\
\hline Mothers & & 154.9 \\
Mean height $(\mathrm{cm})$ & 154.7 & 67.4 \\
Mean weight $(\mathrm{kg})$ & 66.7 & 28.1 \\
Mean body mass index & 27.9 & 56 \\
\% overweight* & 49 & 16.9 \\
Children & & 14.5 \\
\% short staturet & 9.3 & 14.2 \\
\hline
\end{tabular}

"Body mass index above 85th percentile for U.S. population. †Height below 5 th percentile for U.S. population.

¥Weight for height above 95th percentile for U.S. population. Units Conversion Key: $2.54 \mathrm{~cm}=1$ inch; $2.20 \mathrm{~kg}=1$ pound.

Although not apparent in our relatively small and homogeneous samples, larger population studies have demonstrated an inverse relationship between socioeconomic status and fatness. Lower income may be related to less employment and therefore reduced energy expenditure at work, increased consumption of inexpensive energy-dense foods, and reduced availability of energy-consuming recreational opportunities, such as sports and gardening. The variables most strongly and positively associated with BMI for the mothers in this study, however, were acculturationrelated factors: a longer residency in the United States and the ability to speak English.

Children's height. Nine percent of the children in Tulare and $17 \%$ of children in San Jose had heights below the 5 th percentile of a standard reference U.S. population of the same age and sex (National Center for Health Statistics). These percentages were lower than those for Hispanic children observed in the 1960s and 1970s, an advance that may reflect improvement in diet and health. However, the children studied were still slightly shorter than a representative sample of contemporary U.S. Hispanics (HHANES), indicating that environmental influences may hinder this very low-income segment of the Hispanic population from achieving its genetic potential.

Although neither per capita nor family income were related to children's stature (probably because of the small range in income), other facets of socioeconomic status were found to be predictive of height. For example, taller children tended to come from families with fewer children. An increased number of children results in the distribution of the family's resources, financial and otherwise, among more people. Taller children also tended to have mothers who had completed more years of schooling. Education and income are interrelated, and increased education can have an indirect effect on height by means of increased money available for food and health care. In the Tulare and San Jose samples, in which income was universally low, the education level of the mother may have had an important effect on income utilization.

Even after adjusting for socioeconomic variables, the stature of children from Tulare farmworker families was significantly greater than that of the San Jose children. Better access to free or supplementary food in Tulare may have had an impact on children's growth. Significantly more farmworker families reported obtaining food from gardens, local farms, food banks, relatives and friends and, in the case of school-aged children, free school lunches. The extent to which alternative food sources contributed to the diet of the children in the study is unknown and requires further investigation. Additionally the higher cost of living in San Jose may have resulted in less disposable income available for quality foods and medical care.

A strong relationship was also observed between maternal and child height and weight. While representing a genetic component, this association 
may also reflect multigenerational exposure to similar environmental influences. Furthermore, because heights and weights were not significantly different between mothers in Tulare and San Jose, the maternal measurements do not explain the differences observed in the children.

Although the significance to the individual is unclear, the mean height of a population is often used as a gauge of the average nutritional status of the population. As summarized in a recent report by the United Nations, "It is the factors associated with the process of becoming small, not the state of being small, that are the real concern. ..." In this context, the observed stature deficit, especially in San Jose, appears to reflect inequities in living conditions that should be addressed.

Children's weight. Weight-for-age distributions for the Tulare and San Jose children studied were similar to standards from the National Center for Health Statistics. However, weight adjusted for height was significantly higher for children in this study and was similar to that of other lowincome Hispanic children as reported in recent U.S. studies. The overall prevalence of overweight (weight for height above the 95th percentile) was approximately $14 \%$ among both Tulare and San Jose children, which is significantly higher than the $5 \%$ expected in a nationally representative sample of children.

The relationship between children's weight and socioeconomic and cultural influences is complex. While appropriate weight adjusted for height was not associated with income, it was associated with the receipt of public assistance other than WIC; with having more disposable income (i.e., more household possessions and a lower housing cost); having fewer siblings; and having a mother who had lived in the United States longer.

The problems associated with obesity in children may persist into adulthood. These problems include psychosocial maladjustment, abnormal glucose tolerance, hypertension, and hyperlipidemia. Although measures of fatness are needed to determine with certainty if an overweight child is also overfat, the large number of overweight children in this study is cause for concern.

An intriguing question is how a population of children can at the same time fail to achieve their height potential, which is a reflection of undernutrition, and yet be overweight, a reflection of overnutrition. It has been suggested that a diet characterized by low quality (i.e., lacking in certain nutrients such as quality protein), and high quantity (i.e., excessive calories) could be responsible. An inadequate diet in the early years or during critical growth periods followed by an overadequate diet thereafter is another possible explanation.

\section{Conclusion}

Despite the secular gains in height recorded for Hispanic children since the 1960s and 1970s, there remains among this very low-income segment of the Hispanic population a high prevalence of short stature. This stature deficit, while perhaps of little consequence to the individual, is at least in part a reflection of the social and economic disadvantages experienced by this population. The high prevalence of overweight in both children and adult women in the study may be of more concern because of the potential health risks to the individual as well as health costs to society. Even after adjusting for the socioeconomic variables that differed between the Tulare and San Jose samples, and the body measurements of the mothers to account for hereditary differences, there remained a significant deficit in the stature of the San Jose children. Whether this difference is due to sample selection (because volunteers were not randomly selected), activity patterns, or availability of food or other resources has yet to be determined.

To evaluate the prevalence of overweight and short stature, this study used height and weight standards for the U.S. population, as was done for Hispanic HANES. The findings might have been different if standards for Mexican populations had been used. The issue of genetic potential in height

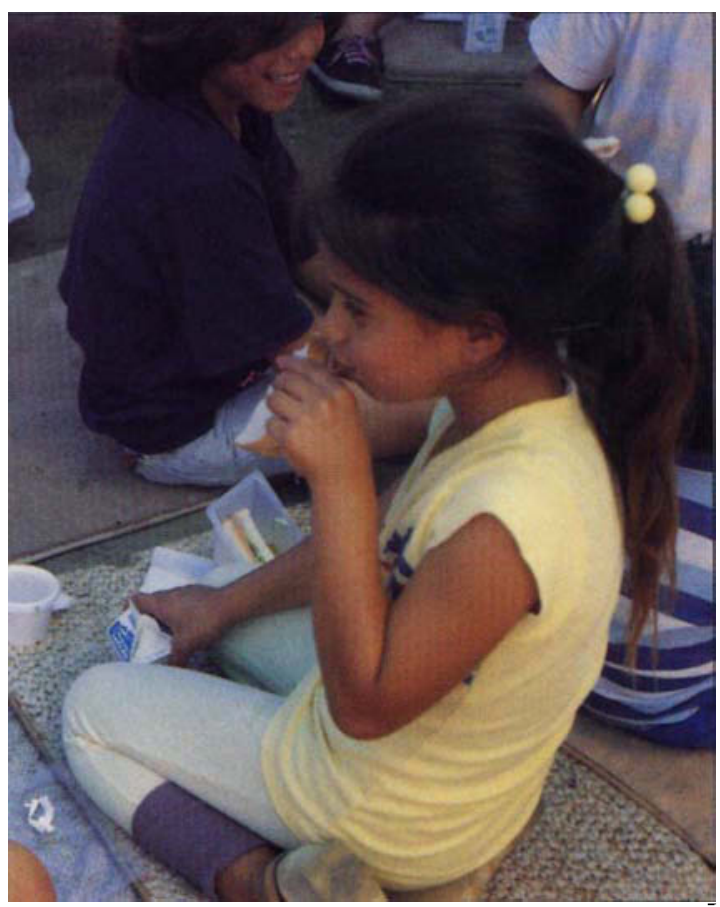

Hispanic children from low-income families were shorter and more overweight than a national sample of Hispanics. Poor diet may affect their growth.

for Hispanics is particularly controversial and deserves further study.

It must be emphasized that although the primary objective of this analysis was to examine the nutritional status of low-income groups of Hispanic women and children using height and weight measurements, the effects of nutrition cannot be separated from other socioeconomic factors. Future analyses are needed which focus on dietary factors and the presence of illnesses, as well as socioeconomic factors, such as acculturation, which may affect growth and overweight among low-income Hispanics. At that point, appropriate, culturally sensitive public health measures can be instituted.

L.D. Ritchie is a doctoral candidate, D.H. Calloway is Professor Emerita, S.P. Murphy is Adjunct Associate Professor, and J.P. Ikeda is Nutrition Education Specialist, all in the Department of Nutritional Sciences, UC Berkeley; O. Receveur is Assistant Professor, McGill University, Quebec; and C.L. Lamp is Home Economist, UC Cooperative Extension, Tulare County. 